\title{
Flows along arch filaments observed in the GRIS 'very fast spectroscopic mode'
}

\author{
S. J. González Manrique ${ }^{1,2}$, C. Denker ${ }^{1}$, C. Kuckein ${ }^{1}$, A. Pastor
} Yabar $^{3,4}$, M. Collados ${ }^{3}$, M. Verma ${ }^{1}$, H. Balthasar ${ }^{1}$, A. Diercke ${ }^{1,2}$, C. E. Fischer ${ }^{5}$, P. Gömöry ${ }^{6}$, N. Bello González ${ }^{5}$, R. Schlichenmaier ${ }^{5}$, M. Cubas Armas ${ }^{3,4}$, T. Berkefeld ${ }^{5}$, A. Feller ${ }^{5}$, S. Hoch ${ }^{7}$,

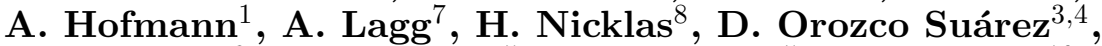
D. Schmidt ${ }^{9}$, W. Schmidt ${ }^{5}$, M. Sigwarth ${ }^{5}$, M. Sobotka ${ }^{10}$, S. K. Solanki ${ }^{7,11}$, D. Soltau ${ }^{5}$, J. Staude ${ }^{1}$, K. G. Strassmeier ${ }^{1}$, R. Volkmer ${ }^{5}$, O. von der Lühe ${ }^{5}$ and T. Waldmann ${ }^{5}$

${ }^{1}$ Leibniz-Institut für Astrophysik Potsdam, 14482 Potsdam, Germany email: smanrique@aip.de

${ }^{2}$ Universität Potsdam, Institut für Physik and Astronomie, 14476 Potsdam, Germany

${ }^{3}$ Instituto de Astrofísica de Canarias, 38205 La Laguna, Tenerife, Spain

${ }^{4}$ Departamento de Astrofísica, Universidad de La Laguna, 38205 La Laguna, Tenerife, Spain

${ }^{5}$ Kiepenheuer-Institut für Sonnenphysik, 79104 Freiburg, Germany

${ }^{6}$ Astronomical Institute, Academy of Sciences, 05960 Tatranská Lomnica, Slovak Republic

${ }^{7}$ Max-Planck-Institut für Sonnensystemforschung, 37077 Göttingen, Germany

${ }^{8}$ Institut für Astrophysik, Georg-August-Universität, 37077 Göttingen, Germany

${ }^{9}$ National Solar Observatory, Sacramento Peak, Sunspot, NM 88349, USA

${ }^{10}$ Astronomical Institute, Academy of Sciences, 25165 Ondřejov, Czech Republic

${ }^{11}$ School of Space Research, Kyung Hee University, Yongin, Gyeonggi-Do, 446-701, Korea

\begin{abstract}
A new generation of solar instruments provides improved spectral, spatial, and temporal resolution, thus facilitating a better understanding of dynamic processes on the Sun. High-resolution observations often reveal multiple-component spectral line profiles, e.g., in the near-infrared He I $10830 \AA$ triplet, which provides information about the chromospheric velocity and magnetic fine structure. We observed an emerging flux region, including two small pores and an arch filament system, on 2015 April 17 with the 'very fast spectroscopic mode' of the GREGOR Infrared Spectrograph (GRIS) situated at the 1.5-meter GREGOR solar telescope at Observatorio del Teide, Tenerife, Spain. We discuss this method of obtaining fast (one per minute) spectral scans of the solar surface and its potential to follow dynamic processes on the Sun. We demonstrate the performance of the 'very fast spectroscopic mode' by tracking chromospheric high-velocity features in the arch filament system.
\end{abstract}

Keywords. Sun: chromosphere, Sun: photosphere, Sun: filaments, Sun: infrared, methods: data analysis, techniques: spectroscopic

\section{Introduction}

The emergence of magnetic flux (Zwaan 1985) in the form of rising $\Omega$-loops is characterized by strong photospheric and chromospheric downflows near footpoints, coalescence of small-scale magnetic features, and dark fibrils within the arch filament system (AFS) with upward motions connecting opposite-polarity patches as well as abnormal granulation and transverse magnetic fields between these patches. The horizontal proper motions 
were studied, for example, by Strous et al. (1996) and Verma et al. (2016) using feature and local correlation tracking (LCT, Verma \& Denker 2011). Investigating strong chromospheric absorption lines, e.g., the He I $10830 \AA$ triplet reveals supersonic downflows near the footpoints of $\Omega$-loops (e.g., Schmidt \& Schlichenmaier 2000, Lagg et al. 2007, Xu et al. 2010, Balthasar et al. 2016, González Manrique et al. 2016). The size of emerging flux regions (EFRs) reaches from small sunspots, over pores, to micro-pores (González Manrique et al. 2017), which all exhibit similar properties, even though the velocities scale with size. Besides line fitting a variety of spectral inversion methods were employed in these studies to infer photospheric and chromospheric velocities.

At the GREGOR telescope, GRIS (Collados et al. 2012) in the 'very fast spectroscopic mode' (VFSM) achieves a one-minute cadence comparable to that of imaging spectrometers (Denker 2010), covering an area of about $66^{\prime \prime} \times 24^{\prime \prime}$. Even higher cadences become possible by sacrificing spatial and spectral resolution. For example, the Fast Imaging Solar Spectrograph (FISS, Chae et al. 2013) is a dual-beam échelle spectrograph installed at the 1.6-meter New Solar Telescope (NST) at Big Bear Solar Observatory, California. It scans a field-of-view (FOV) of $60^{\prime \prime} \times 60^{\prime \prime}$ in less than $20 \mathrm{~s}$, either in the $\mathrm{H} \alpha$ line at $6562.8 \AA$ or in the Ca II NIR line at 8542.1 $\AA$. Conceptually similar to GRIS but designed as a multi-slit, dual-beam spectropolarimeter, the Facility Infrared Spectropolarimeter (FIRS, Jaeggli et al. 2010) shortens the scanning time by recording spectra with four slits at the same time. FIRS is operated at the 0.76-meter Dunn Solar Telescope (DST) at the National Solar Observatory/Sacramento Peak, New Mexico. Both FISS and FIRS share many design concepts with GRIS, and our motivation is to illustrate the potential of GRIS/VFSM in particular and high-cadence, two-dimensional spectroscopy in general.

\section{Observations}

The EFR/AFS was observed with GRIS at the 1.5-meter GREGOR solar telescope (Denker et al. 2012, Kneer 2012, Schmidt et al. 2012) on 2015 April 17 as part of a coordinated observing campaign. González Manrique et al. (2016) described preliminary results of fitting dual-component He I $10830 \AA$ spectral profiles. Complementary data were taken with the GREGOR Fabry-Pérot Interferometer (GFPI, Puschmann et al. 2012) using the photospheric Fe I $6302 \AA$ line and with the CRisp Imaging Spectro-Polarimeter (CRISP, Scharmer et al. 2008) situated at the Swedish Solar Telescope (SST, Scharmer et al. 2003) at Observatorio del Roque de los Muchachos, La Palma, Spain using the photospheric Fe I $6173 \AA$ and chromospheric Ca II $8542 \AA$ lines.

GRIS was operated in the spectroscopic mode covering an 18 - $\AA$-wide spectral region containing the photospheric Si I $10827 \AA$, Ca I $10834 \AA$, and Ca I $10839 \AA$ lines as well as the chromospheric He I triplet at $10830 \AA$. The dispersion was $18.03 \mathrm{~m} \AA$ pixel ${ }^{-1}$. The pixel size along the slit and spatial step size were almost identical, i.e., $0.136^{\prime \prime}$ and $0.134^{\prime \prime}$, respectively. Thus, the spectroscopic scans with 180 steps covered a FOV of about $66.3^{\prime \prime} \times 24.1^{\prime \prime}$. A single exposure of $100 \mathrm{~ms}$ duration was recorded at each spatial step, and it took about $58 \mathrm{~s}$ to complete the scan. The observing sequence continued for about one hour and resulted in 64 data cubes. The GREGOR Adaptive Optics System (GAOS, Berkefeld et al. 2012) provided real-time image correction. The rotation compensation of the alt-azimuthal mount (Volkmer et al. 2012) was not yet available so that the FOV turned by $22.3^{\circ}$, which was corrected in post-processing, whereas the image rotation of $0.4^{\circ}$ in one map was negligible. 


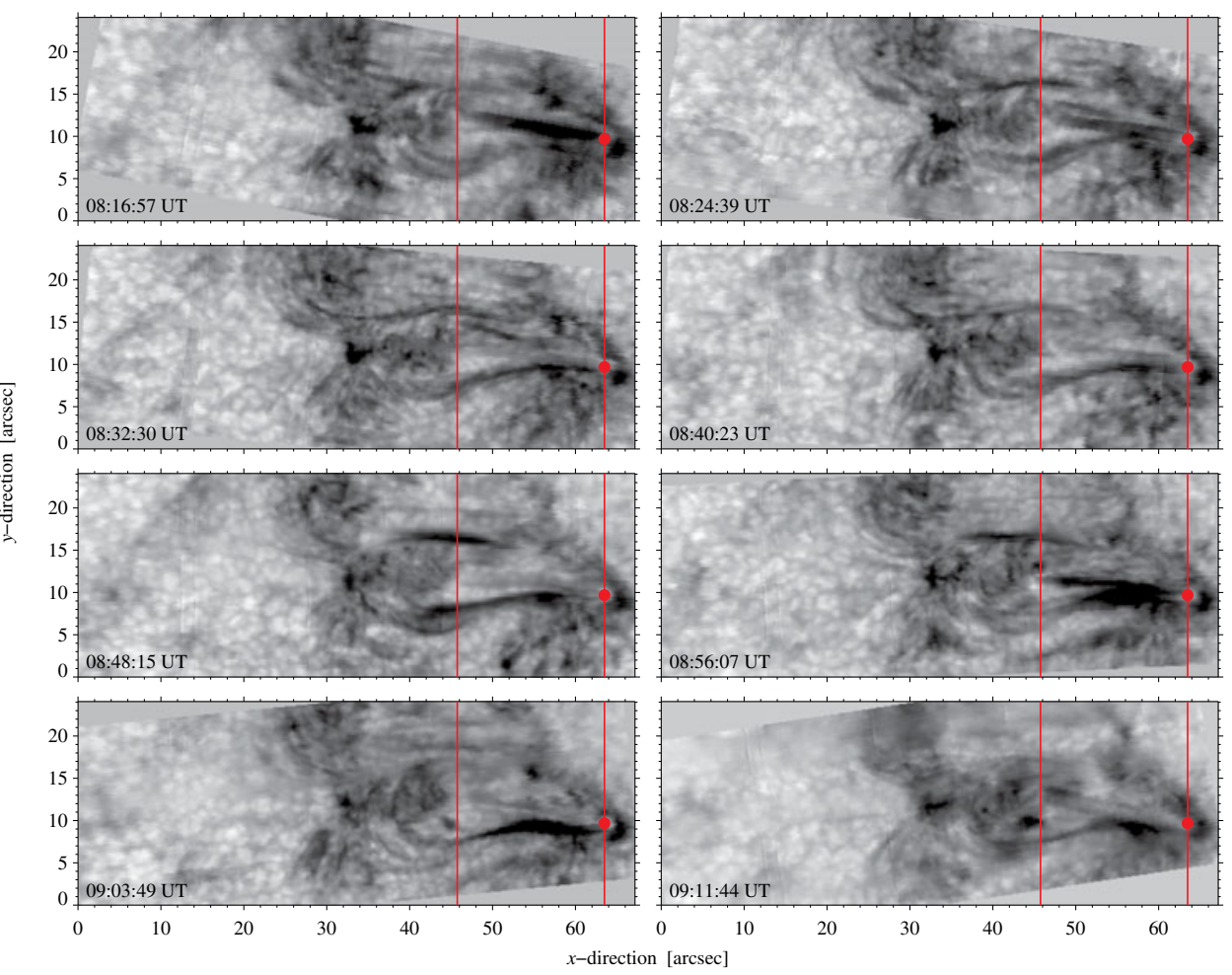

Figure 1. Time-series of eight slit-reconstructed line-core intensity images of the He I triplet's red component. The time interval between successive images is about 8 min. Positions of the space-time diagrams (Figs. 2 and 3) are indicated by vertical lines, and the filled circles refer to the location of a strong downflow kernel with dual-component He I profiles (see Fig. 4).

\section{Results}

The fast cadence of GRIS/VFSM offers access to the highly dynamic chromosphere, which is monitored in this study using the red component of the He I $10830 \AA$ triplet. The time-series of slit-reconstructed He I line-core intensity images in Fig. 1 affirms the good to very good seeing conditions and demonstrates the stable performance of the AO system. Moments of mediocre seeing conditions are rare but become more frequent towards the end of the time-series. They appear in the line-core intensity maps as elongated stripes with low contrast and correspond typically to time intervals of about $10 \mathrm{~s}$. However, for most of the time, the granulation pattern and chromospheric He I absorption features with sub-arcsecond fine structure are very prominently visible.

Spectral scan No. 33 at 08:48:15 UT (fifth panel in Fig. 1) serves as reference for alignment and rotation correction of all spectral data cubes. Light gray areas indicate missing information in the region-of-interest (ROI), which contains two pores of opposite magnetic polarity, one in the center of the ROI at the AO lock-point and the other one at the right edge of the ROI. New magnetic flux is still emerging, which leads to an AFS connecting both pores and other small-scale magnetic elements with multiple dark filament strands. Individual arch filaments change significantly on time-scales of minutes or less, while the overall morphology of the EFR/AFS remains fairly constant over the observing period. Time-lapse movies reveal motions along the fibrils, which are consistent with continuously emerging $\Omega$-loops, where cool material rises to chromospheric heights and beyond at the loop tops and then drains towards the footpoints of the loops. 

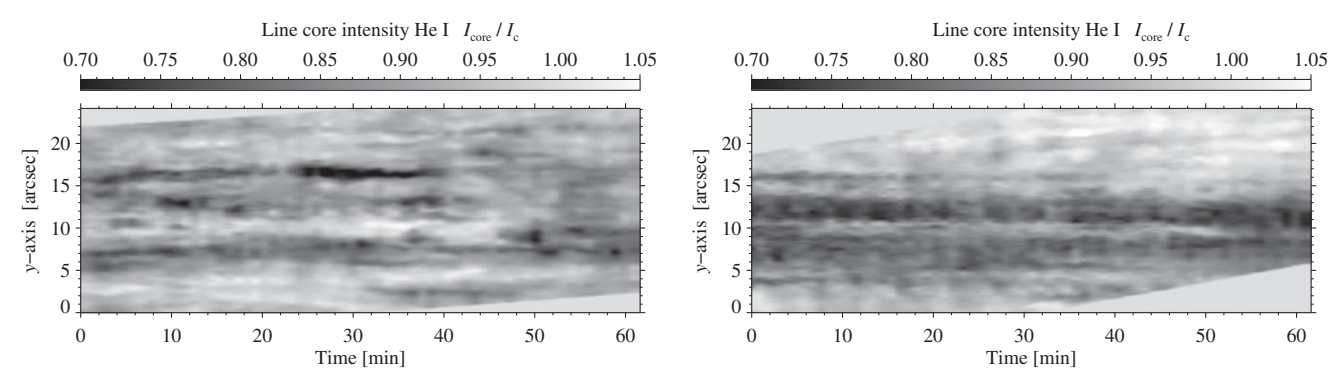

Figure 2. Space-time diagram of the He I line-core intensity $I_{\text {core }}$ (normalized to the continuum intensity $I_{\mathrm{c}}$ ) taken at the footpoint (right) and loop top (left) of the AFS. The positions are marked in Fig. 1 by vertical lines.
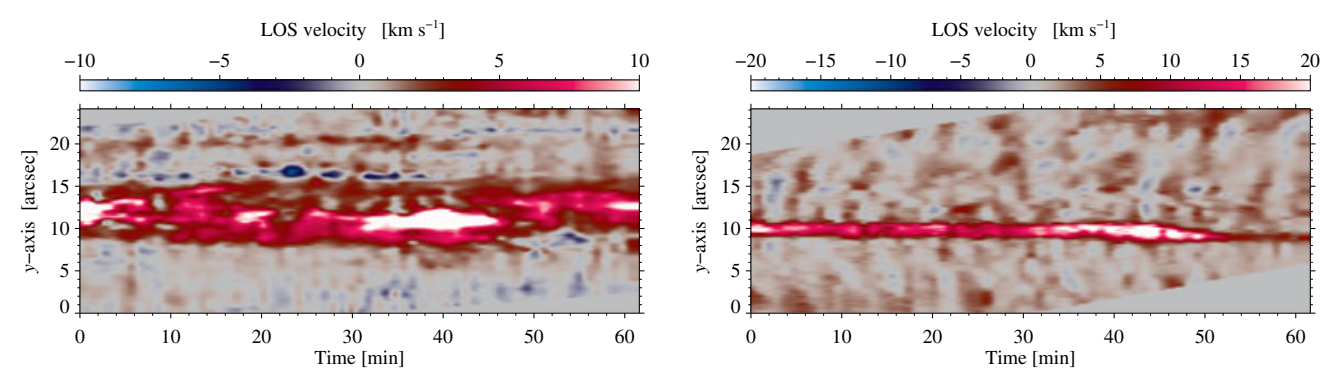

Figure 3. Space-time diagram of the He I LOS velocity (single-component fit) taken at the footpoint (right) and loop top (left) of the AFS. The positions are marked in Fig. 1 by vertical lines.

The most interesting features in the ROI are the loop tops and footpoints of the arch filaments. Two vertical lines in all panels of Fig. 1 mark the positions, where space-time diagrams display the temporal evolution of the He I line-core intensity and line-of-sight (LOS) velocity in Figs. 2 and 3, respectively. Again, light gray areas represent missing values. The majority of LOS velocities results from single-component fits to the observed line profiles unless dual-component fits are more appropriate. In this case, the LOS velocity refers to the fast component. The left panel of Fig. 2 tracks the rising loop top of a thin dark fibril $\left(y \approx 17^{\prime \prime}\right)$, which becomes darkest about $30 \mathrm{~min}$ after the start of the time-series and abruptly vanishes after just over $40 \mathrm{~min}$. A second, more persistent footpoint of a different loop exists at $y \approx 8^{\prime \prime}$, which shows more substructure streaming rapidly past the crosswise line. The associated LOS velocities (left panel of Fig. 3) of the former fibril show upflows approaching $5 \mathrm{~km} \mathrm{~s}^{-1}$, which are typical for a rising $\Omega$ loop, whereas the latter absorption feature near the footpoint of a loop exhibits strong variations of downflows in excess of $10 \mathrm{~km} \mathrm{~s}^{-1}$. The right panels of Figs. 2 and 3 are dedicated to a small kernel with a diameter of $2-3^{\prime \prime}$, where strong persistent downflows are present exceeding $20 \mathrm{~km} \mathrm{~s}^{-1}$, sometimes even reaching more than $40 \mathrm{~km} \mathrm{~s}^{-1}$. These downflows intensify just before the kernel vanishes at $t \approx 50 \mathrm{~min}$. At this location He I absorption is moderately strong and does not change much in time. This downflow kernel is pointed out by filled circles with diameters of about $1.4^{\prime \prime}$ at coordinates $\left(63.2^{\prime \prime}, 9.6^{\prime \prime}\right)$.

The temporal evolution of He I spectra within this downflow kernel deserves further scrutiny. In Fig. 4, the time since the start of the time-series is color coded. The displayed spectra arise from 69 locally averaged spectra contained within the filled circles. They were slightly smoothed for better display but still contain some high-frequency oscillations due to fringes in the NIR spectra. The region was specifically selected because of the strong downflows and the presence of dual-component He I spectral profiles. The rainbow 


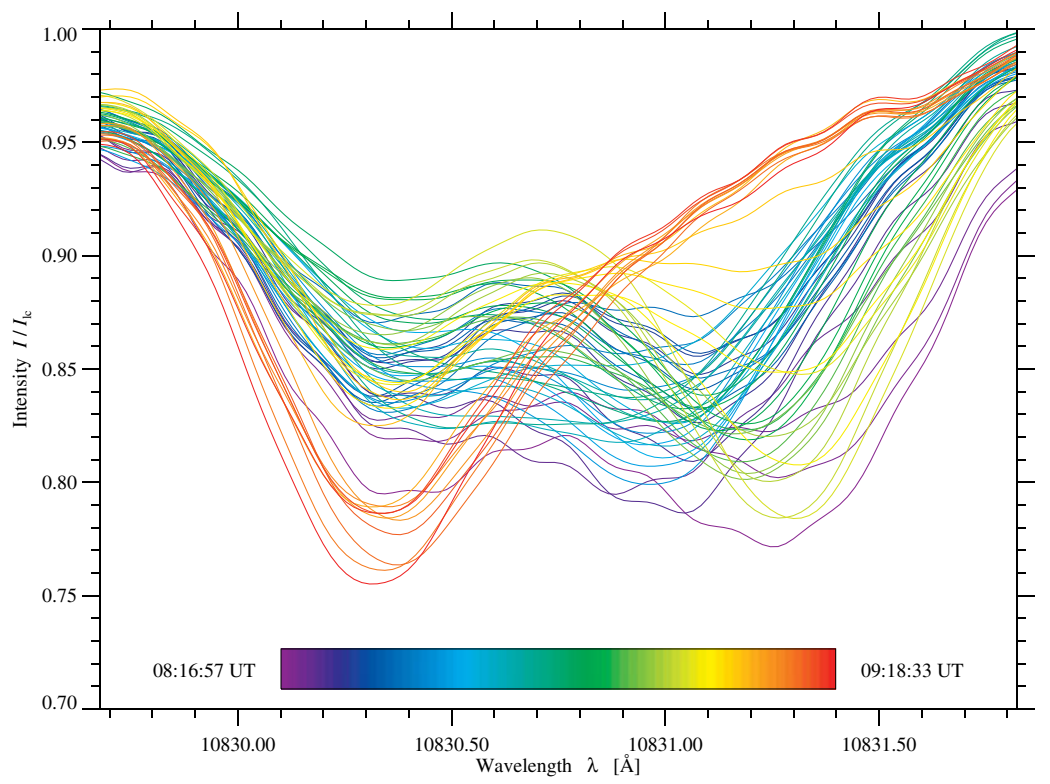

Figure 4. Temporal evolution of spectral profiles (normalized to the local continuum intensity $I_{\mathrm{lc}}$ ) for the red component of the He I triplet. The rainbow-colored bar marks the elapsed time after the start of the one-hour time-series. The spectra were taken at a strong downflow kernel marked by filled circles in Fig. 1. The spectra were slightly smoothed to avoid a cluttered display.

color code immediately reveals time intervals, where the shape of the red component significantly changes. Initially, the slow and fast component form w-shaped profiles with well separated minima of roughly the same intensity. Their line-core intensities are in the range $I_{\text {core }} / I_{\mathrm{c}} \approx 0.80-0.85$. At around 08:41 UT, the combined profile of the slow and fast component becomes more 'bathtub'-like, i.e., the minima are no longer well separated. Starting at 08:50 UT, the fast component becomes dominant reaching linecore intensities of $I_{\text {core }} / I_{\mathrm{c}}<0.8$, and the difference in line-core intensity between the slow and fast component can be as large as $\Delta I \approx 0.1$. The transition to single-component profiles begins abruptly at 09:08 UT resulting in profiles with extended wings towards higher wavelengths and in deep line cores of the slow component.

\section{Discussion and conclusions}

The observed supersonic downflows (up to $40 \mathrm{~km} \mathrm{~s}^{-1}$ ) at the footpoints of arch filaments near pores of an EFR are in very good agreement with previous observations by, for example, Lagg et al. (2007) and Xu et al. (2010). However, magnetic field information is obviously missing in VFSM but can be provided in the future with polarimetric GFPI observations (Balthasar et al. 2011). The downflow kernel discussed above has a size of just a few seconds of arc and is located in close proximity to the pore. Dual-component He I profiles with a strong fast component are continuously present in the vicinity of both pores. They are also sporadically encountered at other locations along the dark fibrils of the AFS. The high-cadence GRIS observations reveal significant changes of the spectral line shapes on time-scales of a few minutes inside the downflow kernels.

The VFSM of GRIS reaches a one-minute cadence comparable to imaging spectrometers by removing the dual-beam polarimeter, which increases the photon flux at the detector by a factor of two. Recording just intensity spectra and not their polarization 
state, lowers the scanning time by a factor of four. Finally, not accumulating spectra at each scan position, another factor of $8-10$ can be gained. Thus, GRIS/VFSM operations mirror the FISS performance and are also applicable to FIRS.

We introduced various ways of visualizing the dynamics of EFRs/AFSs: serial maps of physical parameters, space-time diagrams, and sequential spectra of downflow kernels with dual-component He I profiles. We also attempted applying LCT to a sequence of He I line-core intensity maps. However, the fibril structure of the AFS evolves too quickly to be captured inside the sampling window but tracking slower photospheric motions is possible. This article mainly deals with methodical and technical aspects of the VFSM, the detailed scientific analysis is deferred to a forthcoming article, where we will investigate, for example, if the plasma contained in arch filaments, which exhibit supersonic LOS downflows near the footpoints of the AFS, reaches the photosphere.

\section{Acknowledgements}

The 1.5-meter GREGOR solar telescope was built by a German consortium under the leadership of the Kiepenheuer-Institut für Sonnenphysik in Freiburg with the LeibnizInstitut für Astrophysik Potsdam (AIP), the Institut für Astrophysik Göttingen, and the Max-Planck-Institut für Sonnensystemforschung in Göttingen as partners, and with contributions by the Instituto de Astrofísica de Canarias and the Astronomical Institute of the Academy of Sciences of the Czech Republic. SJGM is supported by the Leibniz Graduate School for Quantitative Spectroscopy in Astrophysics. He is also grateful for an IAU travel grant enabling him to attend the symposium. This study is supported by the European Commission's FP7 Capacities Program under Grant Agreement No. 312495.

\section{References}

Balthasar, H., Bello González, N., Collados, M., et al. 2011, ASP-CS, 437, 351

Balthasar, H., Gömöry, P., González Manrique, S. J., et al. 2016, AN, 337, 1050

Berkefeld, T., Schmidt, D., Soltau, D., et al. 2012, AN, 333, 863

Chae, J., Park, H.-M., Ahn, K., et al. 2013, Solar Phys., 288, 1

Collados, M., López, R., Páez, E., et al. 2012, AN, 333, 872

Denker, C. 2010, $A N, 331,648$

Denker, C., von der Lühe, O., Feller, A., et al. 2012, AN, 333, 810

González Manrique, S. J., Kuckein, C., Pastor Yabar, A., et al. 2016, AN, 337, 1057

González Manrique, S. J., Bello González, N., \& Denker, C. 2017, A\&\&A, accepted

Jaeggli, S. A., Lin, H., Mickey, D. L., et al. 2010, MemSAIt, 81, 763

Kneer, F. 2012, AN, 333, 790

Lagg, A., Woch, J., Solanki, S. K., \& Krupp, N. 2007, A\& $A, 462,1147$

Puschmann, K. G., Denker, C., Kneer, F., et al. 2012, AN, 333, 880

Scharmer, G. B., Bjelksjo, K., Korhonen, T. K., et al. 2003, Proc. SPIE, 4853, 341

Scharmer, G. B., Narayan, G., Hillberg, T., et al. 2008, ApJ, 689, L69

Schmidt, W., von der Lühe, O., Volkmer, R., et al. 2012, AN, 333, 796

Schmidt, W. \& Schlichenmaier, R. 2000, A\&SA, 364, 829

Strous, L., Scharmer, G., Tarbell, T. D., et al. 1996, A\&A, 306, 947

Verma, M., Denker, C., Balthasar, H., Kuckein, C., et al. 2016, A\&\&A, 596, A3

Verma, M. \& Denker, C. 2011, A\& $A$, 529, A153

Volkmer, R., Eisenträger, P., Emde, P., et al. 2012, AN, 333, 816

Xu, Z., Lagg, A., \& Solanki, S. K. 2010, A\&A, 520, A77

Zwaan, C. 1985, Solar Phys., 100, 397 